06

\title{
Влияние лазерного разрушения на нелинейно-оптические свойства core/shell наностержней $\mathrm{Au} / \mathrm{SiO}_{2}$
}

\author{
() Т.А. Чевычелова, А.И. Звягин \\ Воронежский государственный университет, \\ 394018 Воронеж, Россия \\ ฯ e-mail: andzv92@yandex.ru \\ Поступила в редакцию 03.08.2021 г. \\ В окончательной редакции 16.08.2021 г. \\ Принята к публикации 20.08.2021 г.
}

\begin{abstract}
Проанализированы механизмы нелинейно-оптического отклика от ансамблей золотых наностержней, покрытых оболочкой из диоксида кремния $\left(\mathrm{Au} / \mathrm{SiO}_{2} \mathrm{HC}\right)$. Методом $Z$-сканирования под действием $10 \mathrm{~ns}$ импульсов второй гармоники YAG: $\mathrm{Nd}^{3+}$-лазера $(532 \mathrm{~nm})$ найдены зависимости, характерные для нелинейного поглощения и отрицательной динамической линзы. Показано, что усиливающееся с ростом энергии импульса просветление коллоидного раствора вызвано лазерным разрушением $\mathrm{Au} / \mathrm{SiO}_{2} \mathrm{HC}$, реализующимся за счет плавления, что подтверждается теоретическими оценками. Отрицательная нелинейная рефракция отнесена к отклику от сферических наночастиц (Аu НЧ), сформированных при лазерном разрушении НС.
\end{abstract}

Ключевые слова: наностержни золота, core-shell системы, нелинейная рефракция, нелинейное поглощение, $Z$-сканирование.

DOI: $10.21883 / \mathrm{OS} .2021 .12 .51746 .2608-21$

\section{Введение}

Наночастицы (НЧ) благородных металлов проявляют уникальные нелинейно-оптические свойства благодаря особому механизму взаимодействия с лазерным излучением, связанному с существованием коллективных электронных возбуждений, называемых в литературе локализованными или поверхностными плазмонами, время жизни которых ограничено и определяется электронэлектронной $\left(\sim 10^{2} \mathrm{fs}\right)$ и электрон-фононной релаксацией (до $10^{2} \mathrm{ps}$ ) [1-5].

Для плазмонных НЧ сферической формы [1,2,6-9] наблюдают проявления нелинейного поглощения лазерных импульсов. Методом $Z$-сканирования наблюдают профили нелинейного отклика, характерные для обратного насыщенного поглощения (reverse saturable absorption, RSA) у нанокластеров, и просветления или насыщения поглощения (saturable absorption, SA) у более крупных НЧ. По данным [7,8], SA связано с электронными переходами на уровни, лежащие выше уровня Ферми, а RSA - с поглощением свободными носителями заряда в зоне проводимости плазмонных НЧ. Помимо этого, считается возможным посредством лазерного возбуждения индуцировать переход электронов из глубокой $d$-зоны в $s p$-зону золота $[7,8]$.

Интерпретация нелинейного отклика от НЧ несферической формы сложнее. Структура наностержней (НC) подразумевает существование в спектре экстинкции поперечного и продольного плазмонного резонансов. Интерес представляет нелинейный отклик, связанный с продольным резонансом. Время жизни локализованного плазмона в $\mathrm{Au} \mathrm{HC} \mathrm{составляет} \mathrm{40-120} \mathrm{ps} \mathrm{[10].} \mathrm{Это} \mathrm{зна-}$ чение сопоставимо с временем лазерного превращения наностержней в наносферы 30 ps [11]. При воздействии фемтосекундных лазерных импульсов на длине волны излучения вблизи продольного плазмонного резонанса $(700 \mathrm{~nm}) \mathrm{Au} \mathrm{HC}$ демонстрируют нелинейное поглощение и нелинейную рефракцию положительного знака, что связывают с однофотонным поглощением [12] или с поглощением свободными носителями заряда [13]. Однако и при воздействии наносекундных импульсов отмечен сильный нелинейно-оптический отклик НЧ цилиндрической и пирамидальной геометрии [14-16]. Для треугольных НЧ серебра, демонстрирующих широкий контур просветления на длине волны $532 \mathrm{~nm}$, установлено, что SA связано с их разрушением и превращением в сферы, у которых плазмонный пик находится в области $420 \mathrm{~nm}$ [16]. Это заключение не случайно. В ряде работ отмечено, что действие даже коротких лазерных импульсов приводит к нагреву, плавлению и разрушению НЧ размером несколько десятков нанометров [17-20]. Чтобы избежать деструкции НЧ, интенсивность излучения подбирают ниже порога их разрушения.

Таким образом, наблюдаемые оптические нелинейности, особенно эффекты нелинейного поглощения для плазмонных НЧ, требуют учета вклада в наблюдаемые сигналы процессов их фотодеградации. Обоснование механизма возникновения нелинейно-оптического отклика плазмонных НЧ имеет важное значение для рассмотрения аналогичных свойств в гибридных структурах с плеэкситонным взаимодействием [20,21]. В подобных наноструктурах благодаря взаимодействию между плазмоном в металлической НЧ и возбуждением (например, экситоном в квантовых точках) возможны эффекты, 


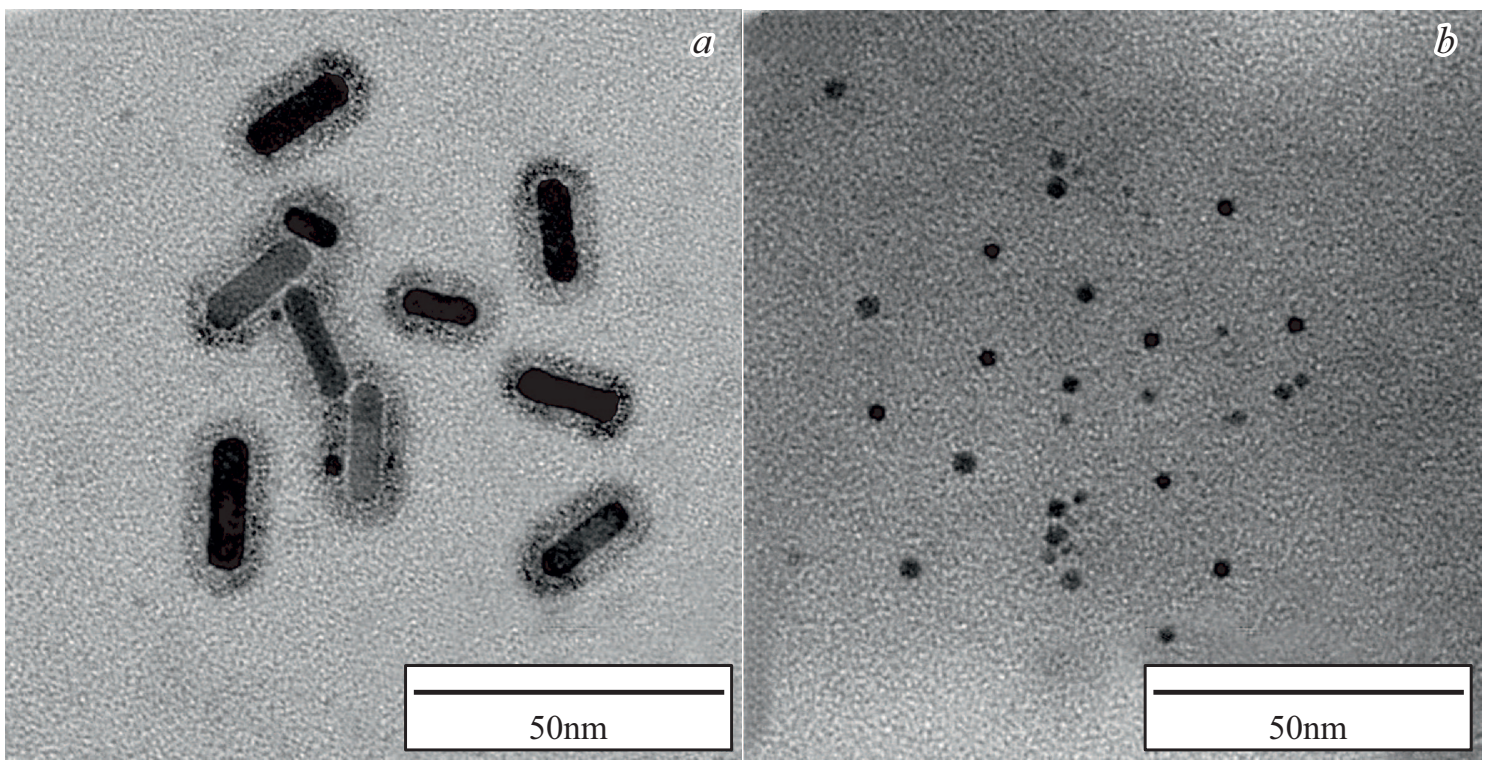

Рис. 1. ПЭМ-изображения до $(a)$ и после $(b)$ воздействия лазерного излучения $\mathrm{Au} / \mathrm{SiO}_{2} \mathrm{HC}$.

вызванные изменением вероятности переходов в одной из компонент плеэкситонных наноструктур. Такие эффекты могут оказывать существенное влияние на оптические нелинейности, имеющие накопительный характер [22-26]. Взаимное расстояние компонентов плеэкситонных наноструктур является одним из ключевых параметров. Управления расстоянием между компонентами системы, как правило, достигают формированием инертных органических или неорганических оболочек. Часто используют $\mathrm{SiO}_{2}$-оболочки. Однако нелинейнооптические свойства core/shell плазмонных наноструктур сферической формы рассматривается лишь в нескольких работах $[27,28]$, а для несферических НЧ - отсутствуют вовсе.

Данная работа частично восполняет этот пробел. Она посвящена анализу нелинейно-оптического отклика коллоидных НЧ золота цилиндрической формы, покрытых оболочкой из диоксида кремния $\left(\mathrm{Au} / \mathrm{SiO}_{2} \mathrm{HC}\right)$, наблюдаемого методом $Z$-сканирования под действием $10 \mathrm{~ns}$ лазерных импульсов на длине волны второй гармоники $\mathrm{Nd}$ :YAG-лазера $\lambda=532 \mathrm{~nm}$.

\section{Экспериментальные методики измерения и приготовления образцов}

Приготовление исследуемых образцов $\mathrm{Au} / \mathrm{SiO}_{2} \mathrm{HC}$ осуществляли в два этапа: выращивание $\mathrm{Au} \mathrm{HC}$ и покрытие готовых наностержней оболочкой из диоксида кремния.

Для формирования $\mathrm{Au} \mathrm{HC}$ использовали многостадийный подход в рамках коллоидного синтеза [20]. Водный раствор поверхностно-активного вещества цетилтриметиламмония бромида (ЦТАБ) используется для выращивания цилиндрических НЧ, являясь одновремен- но слабым восстановителем и анизотропной средой. Затравочный раствор наносфер золота размером $3 \mathrm{~nm}$ получали путем восстановления водной золотохлористоводородной кислоты $\mathrm{HAuCl}_{4}(7 \mu 1,0.36 \mathrm{M})$ раствором $\mathrm{NaBH}_{4}(1.0 \mathrm{ml}, 5 \mathrm{mM})$ в водном растворе ЦТАБ $(20 \mathrm{ml}$, $0.02 \mathrm{mM})$. Анизотропию роста НЧ обеспечивает ростовой раствор, который получали в результате смешивания $\mathrm{HAuCl}_{4}(28 \mu \mathrm{l}, 0.36 \mathrm{M})$, ЦТАБ $(50 \mathrm{ml}, 0.1 \mathrm{mM}), \mathrm{AgNO}_{3}$ $(0.1 \mathrm{ml}, 0.02 \mathrm{M})$ и $\mathrm{C}_{6} \mathrm{H}_{8} \mathrm{O}_{6}(5 \mathrm{ml}, 0.05 \mu \mathrm{M})$. Добавление $\mathrm{AgNO}_{3}$ способствует управлению отношения длины к диаметру $\mathrm{Au}$ НС. Готовый раствор очищали от продуктов реакции путем промывки дистиллированной водой посредством нескольких циклов центрифугированиядиспергирования. По данным ПЭМ, полученные НЧ представляли собой наностержни и имели средний размер $6 \times 18 \mathrm{~nm}$.

На основе полученных $\mathrm{Au}$ HC создавали core/shell структуры путем наращивания слоя диоксида кремния [29]. Формирование оболочки $\mathrm{SiO}_{2}$ на поверхности $\mathrm{Au} \mathrm{HC}$ осуществляли путем функционализации поверхности $\mathrm{Au} \mathrm{HC}$ монослоем (3-меркаптопропил) триметоксисилан (3-MPTMS) с последующим формированием плотных слоев $\mathrm{SiO}_{2}$ метасиликатом натрия $\left(\mathrm{Na}_{2} \mathrm{O}\left(\mathrm{SiO}_{2}\right)\right)$ [21]. Для этого $0.5 \mathrm{ml}$ гидролизованного $2 \mathrm{mM}$ раствора 3-MPTMS смешивали с $20 \mathrm{ml}$ раствора коллоидных $\mathrm{Au} \mathrm{HC}(\mathrm{pH}=9.0)$. Далее в реакционную смесь добавляли $4 \mathrm{ml}$ водного раствора $\mathrm{Na}_{2} \mathrm{SiO}_{3}$ $(0.38 \mathrm{mM})$, регулируя $\mathrm{pH}$ до значения 10.2 , и оставляли перемешивать в течении 7 дней. Размер полученной оболочки составлял $5 \mathrm{~nm}$ (рис. 1, $a$ ).

Размеры $\mathrm{Au} / \mathrm{SiO}_{2} \mathrm{HC}$ и толщину оболочки $\mathrm{SiO}_{2}$ на ее поверхности определяли с помощью просвечивающего электронного микроскопа (Libra 120, Carl Zeiss, Германия). Исследование спектров экстинкции света 


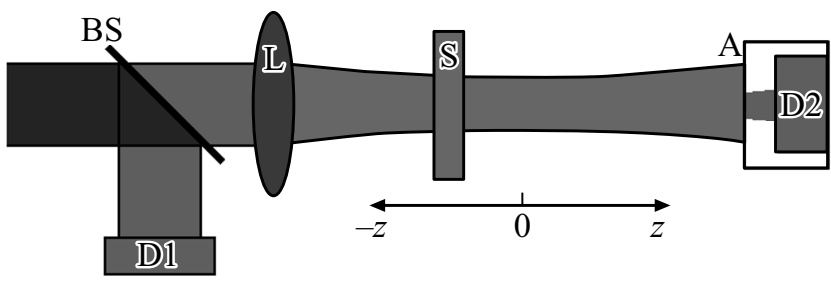

Рис. 2. Схема Z-сканирования: $\mathrm{BS}$ - светоделитель; L линза; D1 - первый фотодиод; S - образец; А - диафрагма; D2 - второй фотодиод.

синтезированными ансамблями $\mathrm{Au} / \mathrm{SiO}_{2} \mathrm{HC}$ осуществляли с использованием спектрометра USB2000+ (Ocean Optics) с источником излучения USB-DT (Ocean Optics).

Для исследования нелинейно-оптических свойств исследуемых образцов использовали метод Z-сканирования. Он основан на измерении нормализованного пропускания исследуемого образца при его перемещении относительно фокальной плоскости собирающей линзы, вдоль оптической оси $z$ [30]. Коэффициент нормализованного пропускания определяется как отношение коэффициента пропускания образца $T_{\text {meas }}(z)$ в точке с координатой $z$ к пропусканию образца в линейном режиме при низкой интенсивности, которое регистрируется вдали от фокуса линзы $T_{\text {meas }}(z \rightarrow \infty)$ :

$$
T=\frac{T_{\text {meas }}(z)}{T_{\text {meas }}(z \rightarrow \infty)} .
$$

В этом методе пропускание образца измеряли при условии различного поперечного размера гауссова пучка (различной интенсивности). Другими словами, при изменении координаты $z$ фактически осуществляли сканирование по интенсивности лазерного излучения.

Нормализованное пропускание образцов коллоидных растворов $\mathrm{Au} / \mathrm{SiO}_{2} \mathrm{HC}$ исследовали в геометрии с открытой (позволяет исследовать только абсорбционные нелинейные процессы) апертурой (ОА), а также в геометрии с закрытой апертурой (СА). В последнем случае вклад в нормализованное пропускание $(Z$-скан) вносят еще и рефракционные эффекты. Экспериментальная установка представляла собой установленные на одной оптической оси YAG: $\mathrm{Nd}^{3+}$-лазер (вторая гармоника, $532 \mathrm{~nm}, 10 \mathrm{~ns}$ ), светоделитель (BS), сферическая линза с фокусным расстоянием $300 \mathrm{~mm}(\mathrm{~L})$, за которой установлен образец $\mathrm{S}$ в $5 \mathrm{~mm}$ кварцевой кювете (рис. 2).

\section{Нелинейно-оптические свойства образцов}

На рис. 3, $a$ представлены Z-сканы от ансамблей $\mathrm{Au} / \mathrm{SiO}_{2} \mathrm{HC}$, полученных в геометрии с ОА и энергии лазерного импульса 0.02 и $0.16 \mathrm{~mJ}$. Для значения энергии лазерного импульса $0.02 \mathrm{~mJ}$ наблюдали увеличение нормализованного пропускания по мере приближения образца к фокальной плоскости линзы $T_{\max }=1.07$. С увеличением энергии импульса до $0.16 \mathrm{~mJ}$ незначительный рост просветления образца до 1.08 сопровождался возникновением симметричного провала $\left(T_{\min }=0.90\right)$ относительно фокальной плоскости линзы $(Z=0)$. Следует отметить, что для $\mathrm{Au} \mathrm{HC}$, синтезированных тем же способом, наблюдали аналогичные $Z$-сканы, что указывает на отсутствие вклада оболочки $\mathrm{SiO}_{2}$ в регистрируемый нелинейный отклик.

Наблюдаемое просветление может быть обусловлено как насыщением плазмонного поглощения, так и падением оптической плотности на длине волны зондирующих импульсов (рис. 3). Падение оптической плотности может быть связано с лазерным разрушением $\mathrm{Au} / \mathrm{SiO}_{2} \mathrm{HC}$. В пользу этого заключения свидетельствует также отсутствие просветления $\mathrm{Au} / \mathrm{SiO}_{2} \mathrm{HC}$ при повторном zсканировании уже облучённого образца. В этом случае наблюдается только сигнал затемнения (рис. 3, a). Наведенное затемнение, возникающее по мере приближения образца к фокальной плоскости линзы, может возникать по нескольким причинам. Наряду с маловероятным механизмом обратного насыщения поглощения (RSA) [7,8] вероятно возникновение фотоиндуцированного рассеяния [16,21]. Аналогично указанным работам фотоиндуцированное рассеяние подтверждено в экспериментах, в которых использовали дополнительный фотодиод, установленный в используемой схеме $Z$-сканирования, под углом $5^{\circ}$ к оптической оси. В этой схеме регистрировали сигнал рассеяния, который в $Z$-скане представлял собой симметричный пик с максимумом при $z=0$.

Анализ результатов, полученных с помощью ПЭМ и спектров экстинкции света образцов до и после воздействия теми же лазерными импульсами, что использовали при $Z$-сканировании, свидетельствует о фотодеструкции $\mathrm{Au} / \mathrm{SiO}_{2}$ НС. Согласно данным ПЭМ, после экспонирования коллоидного раствора $\mathrm{Au} / \mathrm{SiO}_{2} \mathrm{HC}$ микрофотографии содержат только сферические наночастицы размером 2-4 nm (рис. $1, b)$. Это заключение согласуется с данными работ $[19,20]$, в которых наблюдали разрушение $\mathrm{Au} \mathrm{HC}$ в поле наносекундных импульсов вследствие тепловых процессов. Полученные данные свидетельствуют о том, что $\mathrm{Au} / \mathrm{SiO}_{2} \mathrm{HC}$ неустойчивы, так же как и $\mathrm{Au} \mathrm{HC}$ в поле лазерных импульсов. В этом состоит отличие от случая сферических core/shell $\mathrm{Ag} / \mathrm{SiO}_{2} \mathrm{HЧ}$, когда оболочка $\mathrm{SiO}_{2}$ предотвращала фотодеструкцию $\mathrm{HЧ}$ серебра $\mathrm{Ag} / \mathrm{SiO}_{2}$ [28].

В спектрах экстинкции света образцов после их экспонирования коллоидного раствора $\mathrm{Au} / \mathrm{SiO}_{2} \mathrm{HC}$ теми же лазерными импульсами полностью исчезал пик при $636 \mathrm{~nm}$, обусловленный продольным плазмонным резонансом. Этот факт также свидетельствует об изменении формы НЧ с цилиндрической на сферическую. Кроме того, пик при $534 \mathrm{~nm}$ смещается к $508 \mathrm{~nm}$, и поглощение на длине волны $532 \mathrm{~nm}$ также уменьшается, что и определяет просветление (рис. 4).

Для детализации механизма лазерного разрушения золотых наностержней обратимся к структурным дан- 
$a$

$b$
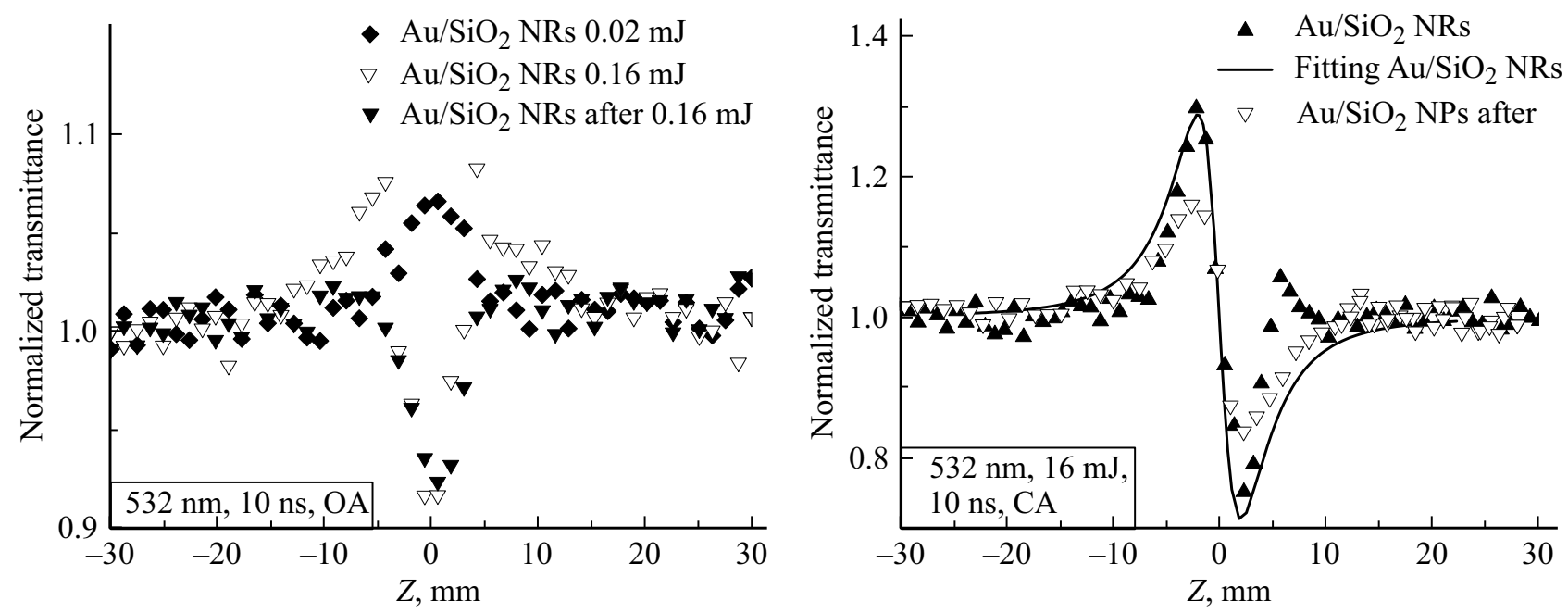

Pис. 3. $Z$ - сканы $\mathrm{Au} / \mathrm{SiO}_{2} \mathrm{HC}$, полученные в геометрии с открытой $(a)$ и закрытой $(b)$ апертурой.

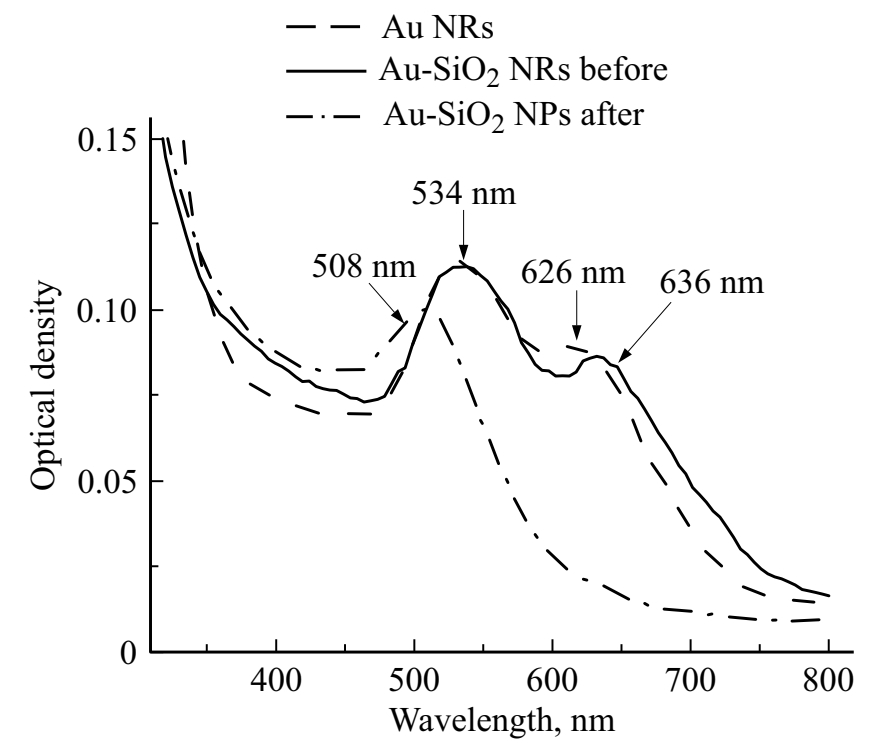

Рис. 4. Спектры экстинкции, демонстрирующие фотоиндуцированное преобразование $\mathrm{Au} \mathrm{HC}$ в Au NPs.

ным. На ПЭМ изображениях (рис. 1,a) для $\mathrm{Au} / \mathrm{SiO}_{2}$ НС видна закономерная боковая огранка. Ввиду малой толщины на их фоне видна структура аморфной пленки углерода, на которой они находятся. Дифракционный контраст в пределах отдельных стержней не выявляет наличия границ зерен, тонких двойниковых прослоек, ростовых дислокаций, что свидетельствует о совершенной структуре $\mathrm{Au} / \mathrm{SiO}_{2} \mathrm{HC}$. В таком случае лазерно-индуцируемая рекристаллизация маловероятна. Для длительностей лазерных импульсов, составляющих наносекунды, характерным механизмом преобразования геометрии НЧ в таком случае может быть их плавление [17].
В пользу лазерного плавления $\mathrm{Au} / \mathrm{SiO}_{2} \mathrm{HC}$ свидетельствуют также численные оценки достигаемых температур в рамках однотемпературной модели нагрева (OTM) [18]. Данная модель применима для наносекундных импульсов, так как за время одного импульса температура возбужденных горячих электронов и решеточной подсистемы успевает эффективно выровняться в каждый момент времени. В таком случае зависимость температуры от времени рассчитывается исходя из уравнения [18]:

$$
\frac{d T}{d t}=\frac{\mu(T)}{\rho C(T)} \Delta T+\frac{Q(t, r)}{\rho C(T)}-j_{D} S_{0}+\frac{3 L}{r_{e f} C(T)} \frac{d r_{e f}}{d t}
$$

где $T(t, r)$ - температура решетки, $\mu(T)$ - теплопроводность золота, $C(T)$ - удельная теплоемкость, $\rho-$ плотность золота, $\Delta$ - оператор Лапласа, $Q(t, r)-$ тепловой источник, $j_{D}$ - отток тепла в окружающую среду, $S_{0}$ - площадь поверхности источника, $L-$ удельная теплота испарения, $r_{e f}=\sqrt[3]{\frac{3 V}{4 \pi}}-$ эффективный радиус наночастицы, $V$ - объем наночастицы. Здесь в первом слагаемом учитывается форма наночастицы, во втором - ее однородный нагрев, в третьем - отток тепла в окружающую среду и в четвертом - испарение электронов с поверхности наностержней. В случае длительных наносекундных импульсов происходит однородный нагрев всего объема наночастицы, и ее форма не оказывает влияние на динамику нагрева. С другой стороны, оболочка из диоксида кремния должна предотвращать испарение электронов. Это дает возможность упростить решение уравнения следующим образом. Вопервых, произвести интегрирование не по цилиндрическому, а по сферическому объему, вводя эффективный радиус эквивалентной сферической наночастицы. Вовторых, пренебречь испарением с поверхности. Таким 


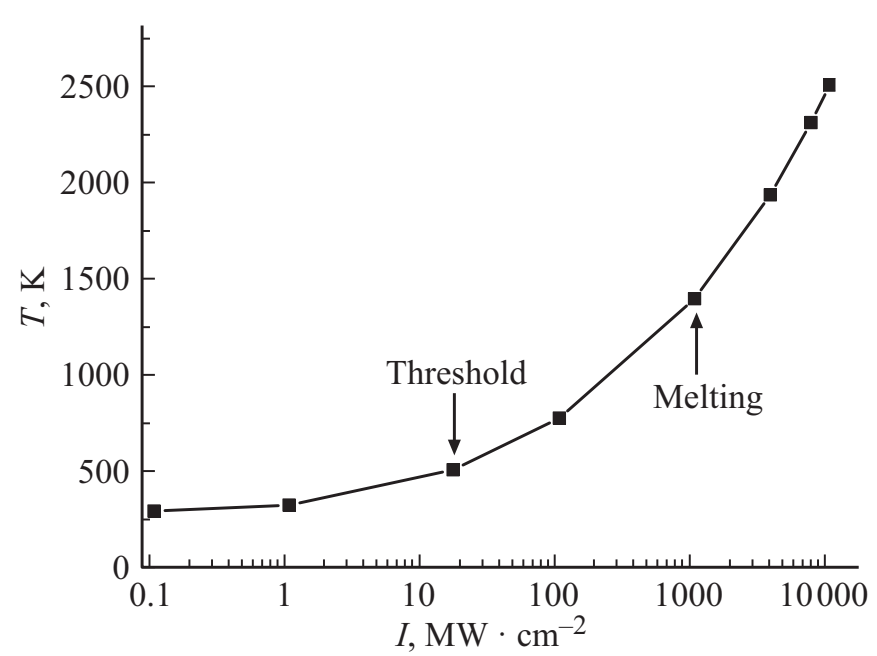

Pис. 5. Зависимость температуры от интенсивности зондирующих импульсов для наностержней $\mathrm{Au} / \mathrm{SiO}_{2} \mathrm{HC}$ размером ядра $6 \times 18 \mathrm{~nm}\left(r_{e f}=5 \mathrm{~nm}\right)$.

образом, оставшиеся слагаемые расписываем как в [18]:

$$
\frac{d T}{d t}=\frac{3 K_{a b s} I_{0} f(t)}{4 r_{e f} C(T) \rho}-\frac{\mu_{\infty} T}{(s+1) r_{e f}^{2} C(T) \rho}\left[\left(\frac{T}{T_{\infty}}\right)^{s+1}-1\right],
$$

где $T_{\infty}-$ температура окружающей среды, $K_{a b s}-$ эффективность поглощения излучения НЧ, $I_{0}$ - интенсивность падающего излучения, $f(t)$ - форма импульса $\mu_{\infty}$ - теплопроводность окружающей среды, $s=1-$ степенной показатель, зависящий от окружающей среды (значение указано для воды).

Зависимость температуры НЧ от интенсивности лазерного излучения $T(I)$ представлена на рис. 5. Видно, что при пороговой интенсивности начала просветления $I_{\text {thresh }}=17 \mathrm{MW} \cdot \mathrm{cm}^{-2}$ температура НЧ не достигает температуры плавления $\left(T_{\text {melt }}=1337 \mathrm{~K}\right)$ и составляет порядка $500 \mathrm{~K}$. Однако оценка интенсивности, при которой наступает плавление, составила $I_{m e l t}=930 \mathrm{MW} \cdot \mathrm{cm}^{-2}$. Оказалось, что вблизи фокуса собирающей линзы интенсивности лазерного излучения вполне достаточно для плавления НЧ и изменения их формы с цилиндрической на сферическую, что и определяет наблюдающееся просветление в Z-сканах.

Таким образом, характерным механизмом изменения геометрии $\mathrm{Au} / \mathrm{SiO}_{2} \mathrm{HC}$ в поле лазерных импульсов порядка наносекунд является их плавление с последующим образованием сферических НЧ золота, что проявляется в нелинейно-оптическом отклике в виде просветления коллоидного раствора при воздействии излучения с длиной волны $532 \mathrm{~nm}$.

В случае закрытой апертуры СА исходные $\mathrm{Au} / \mathrm{SiO}_{2} \mathrm{HC}$ и $\mathrm{Au} / \mathrm{SiO}_{2}$ НЧ после фотодеструкции демонстрируют нелинейную рефракцию; кривые $Z$-сканирования для „свежих“ и экспонированных образцов идентичны (рис. $3, b)$. Наличие максимума при отрицательных и минимума при положительных $Z$ указывает на отрицательную нелинейную рефракцию.

Оценку нелинейного показателя преломления выполняли путем аппроксимации кривых $Z$-сканов с помощью формулы [31]:

$$
T=1+\frac{2(-\rho x+2 x-3 \rho)}{\left(x^{2}+9\right)\left(x^{2}+1\right)} \Delta \Phi_{0} .
$$

Здесь $\rho=\beta / 2 k \gamma, x=z / z_{0}, \Delta \Phi_{0}=k \gamma I_{0} L_{e f}-$ сдвиг фаз в фокусе за счет нелинейной рефракции и нелинейного поглощения, $I_{0}-$ интенсивность лазерного излучения в фокусе, $L_{e f}=[1-\exp (-\alpha L)] / \alpha-$ эффективная длина образца, $\alpha$ - линейный коэффициент поглощения, $\gamma$ нелинейный показатель преломления, $\beta$ - коэффициент нелинейного поглощения материалов.

У ансамблей $\mathrm{Au} / \mathrm{SiO}_{2} \mathrm{HC}$ величина нелинейного показателя преломления $\gamma$ составила $-5 \cdot 10^{-15} \mathrm{~cm}^{2} \cdot \mathrm{W}^{-1}$. Оценка вклада тепловой рефракции дала значение коэффициента, равное $\gamma \sim-10^{-17} \mathrm{~cm}^{2} \cdot \mathrm{W}^{-1}$ [30], что на два порядка меньше, чем наблюдаемое в эксперименте для $\mathrm{Au} / \mathrm{SiO}_{2} \mathrm{HC}$. Это позволяет исключить из рассмотрения возможных механизмов рефракции вклад тепловых процессов.

Известно, что для золотых НЧ в процессах поглощения наряду с плазмонным поглощением также важную роль играют межподзонные переходы. В таком случае изменение заселенностей состояний в $d$-зоне и $s p$-зоне в результате поглощения излучения будет сопровождаться изменением нестационарных абсорбционных полос для $\mathrm{Au} / \mathrm{SiO}_{2} \mathrm{HC}$, что в свою очередь приведет к зависимости показателя преломления от интенсивности падающего излучения, в соответствии с дисперсионными соотношениями Крамерса-Кронига [32]. Однако данные о спектральных и энергетических свойствах локализованных состояний в $d$-зоне и $s p$-зоне $[7,8,33]$, а также об их возможности участвовать в переходах, сопровождающихся нелинейной рефракцией, отсутствуют.

Для установления деталей механизма нелинейной рефракции осуществляли построение кривых зависимости нормализованного пропускания от падающей интенсивности, используя данные $Z$-сканов. Это позволило установить пороговые значения интенсивности начала фотодеструкции, ограничения оптической мощности и нелинейной рефракции, равные соответственно 17, 261 и $317 \mathrm{MW} \cdot \mathrm{cm}^{-2}$. Оказалось, что нелинейная рефракция наступает при значительно больших интенсивностях по сравнению с фотодеструкцией золотых наностержней. Таким образом, рефракционный отклик наблюдается преимущественно на сформированных лазерным излучением золотых наносферах Au.

\section{Заключение}

Полученные нелинейно-оптические свойства, спектральные и структурные данные указывают на разрушение core/shell $\mathrm{Au} / \mathrm{SiO}_{2} \mathrm{HC}$ до золотых наносфер в поле 
наносекундных лазерных импульсов с длиной волны $532 \mathrm{~nm}$. Рефракционный отклик наблюдается преимущественно на новообразованных наносферах под действием зондирующего излучения.

\section{Финансирование работы}

Работа поддержана грантом Президента РФ для государственной поддержки ведущих научных школ РФ, проект НШ-2613.2020.2.

\section{Конфликт интересов}

Авторы заявляют, что у них нет конфликта интересов.

\section{Список литературы}

[1] Zhang K., Ganeev R.A., Rao K.S., Maurya S.K., Boltaev G.S., Krishnendu P.S., Yu Z., Yu W., Fu Y., Guo C. // J. Nanomater. 2019. V. 2019 P. 6056528. doi 10.1155/2019/6056528

[2] Mezher M.H., Nady A., Penny R., Chong W.Y., Zakaria R. // Appl. Opt. 2015. V. 54. N 33. P. 9703. doi 10.1364/ao.54.009703

[3] Mezher M.H., Chong W.Y., Zakaria R. // RSC Adv. 2016. V. 6. N 106. P. 104624. doi 10.1039/c6ra22367b

[4] Zhang Y.X., Wang Y.H. // RSC Adv. 2017. V. 7. N 71. P. 45129. doi $10.1039 / \mathrm{c} 7 \mathrm{ra} 07551 \mathrm{k}$

[5] Wurtz G.A., Pollard R., Hendren W., Wiederrecht G.P., Gosztola D.J., Podolskiy V., Zayats A.V. // Nat. Nanotechnol. 2011. V. 6. N 2. P. 107. doi 10.1038/nnano.2010.278

[6] Hua Y., Chandra K., Dam D.H.M., Wiederrecht G.P., Odom T.W. // J. Phys. Chem. Lett. 2015. V. 6. N 24. P. 4904. doi 10.1021/acs.jpclett.5b02263

[7] Mai H.H., Kaydashev V.E., Tikhomirov V.K., Janssens E., Shestakov M.V., Meledina M., Turner S., Van Tendeloo G., Moshchalkov V.V., Lievens P. // J. Phys. Chem. C. 2014. V. 118. N 29. P. 15995. doi 10.1021/jp502294u

[8] Philip R., Chantharasupawong P., Qian H., Jin R., Thomas J. // Nano Lett. 2012. V. 12. N 9. P. 661. doi 10.1021/n1301988v

[9] Sánchez-Dena O., Mota-Santiago P., Tamayo-Rivera L., Crespo-Sosa A., Oliver A., Reyes-Esqueda J.A. // 2013. V. 4. P. 92. doi 10.1364/ome.4.000092

[10] Hu M., Wang X., Hartland G.V., Mulvaney P., Juste J.P., Sader J.E. // J. Am. Chem. Soc. 2003. V. 125. N 48. P. 14925. doi 10.1021/ja037443y

[11] Link S., Burda C., Mohamed M.B., Nikoobakht B., ElSayed M.A. // J. Phys. Chem. A. 1999. V. 103. N 9. P. 1165. doi $10.1021 / \mathrm{jp} 983141 \mathrm{k}$

[12] Gordel M., Kolkowski R., Olesiak-Banska J., Matczyszyn K., Buckle M., Samoc M. // J. Nanophotonics. 2014. V. 9. N 1. P. 093797. doi 10.1117/1.jnp.9.093797

[13] Ban G., Fu X., Peng Z. // Key Eng. Mater. 2014. V. 602-603. P. 993. doi 10.4028/www.scientific.net/KEM.602-603.993

[14] Zheng C., Huang J., Lei L., Chen W., Wang H., Li W. // Appl. Phys. B. 2018. V. 124. N 1. P. 1. doi 10.1007/s00340017-6888-3

[15] Jae Tae Seo, Qiguang Yang, Wan-Joong Kim, Jinhwa Heo, Seong-Min Ma, Austin J., Wan Soo Yun, Sung Soo Jung, Sang Woo Han, Tabibi B., Temple D. // Opt. Lett. 2009. V. 34. N 3. P. 307. doi 10.1364/ol.34.000307
[16] Zvyagin A.I., Perepelitsa A.S., Lavlinskaya M.S., Ovchinnikov O.V., Smirnov M.S., Ganeev R.A. // Optik (Stuttg). 2018. V. 175. P. 93. doi 10.1016/j.ijleo.2018.08.117

[17] Letfullin R.R., George T.F., Duree G.C., Bollinger B.M. // Adv. Opt. Technol. 2008. V. 2008. P. 251718. doi $10.1155 / 2008 / 251718$

[18] Werner D., Furube A., Okamoto T., Hashimoto S. // J. Phys. Chem. C. 2011. V. 115. N 17. P. 8503. doi 10.1021/jp112262u

[19] Attia Y.A., Flores-Arias M.T., Nieto D., Vázquez-Vázquez C., De La Fuente G.F., López-Quintela M.A. // J. Phys. Chem. C. 2015. V. 119. N 23. P. 13343. doi 10.1021/acs.jpcc.5b03046

[20] Гревцева И.Г., Чевычелова Т.А., Дерепко В.Н., Овчинников О.В., Смирнов М.С., Перепелица А.С., Паршина А.C. // Конденсированные среды и межфазные границы. 2021. T. 23. № 1. C. 25. doi $10.17308 / \mathrm{kcmf} .2021 .23 / 3294$

[21] Овчинников О.В., Смирнов М.С., Гревцева И.Г., Дерепко В.Н., Чевычелова Т.А., Леонова Л.Ю., Перепелица А.С., Кондратенко Т.С. // Конденсированные среды и межфазные границы. 2021. Т. 23. № 1. С. 49. doi 10.17308/kcmf.2021.23/3302

[22] Zvyagin A.I., Smirnov M.S., Ovchinnikov O.V. // Optik. 2020. V. 218. P. 165122. doi 10.1016/j.jileo.2020.165122

[23] Kondratenko T.S., Smirnov M.S., Ovchinnikov O.V., Zvyagin A.I., Ganeev R.A., Grevtseva I.G. // Optik. 2020. V. 200. P. 163391. doi 10.1016/j.jileo.2019.163391

[24] Boltaev G.S., Fu D.J., Sobirov B.R., Smirnov M.S., Ovchinnikov O.V., Zvyagin A.I., Ganeev R.A. // Optics Express. 2018. V. 26. N 11. P. 13865. doi 10.1364/OE.26.013865

[25] Кондратенко Т.С., Гревцева И.Г., Звягин А.И., Овчинников О.В., Смирнов М.С. // ОПт. и спектр. 2018. T. 124. № 5. C. 640. doi 10.21883/OS.2018.05.45945.31017; Kondratenko T.S., Grevtseva I.G., Zvyagin A.I., Ovchinnikov O.V., Smirnov M.S. // Opt. Spectrosc. 2018. V. 124. N 5. P. 673. doi 10.1134/S0030400X18050090

[26] Boltaev G.S., Sobirov B., Reyimbaev S., Sherniyozov H., Usmanov T., Smirnov M.S., Ovchinnikov O.V., Grevtseva I.G., Kondratenko T.S., Shihaliev H.S., Ganeev R.A. // Appl. Phys. A. 2016. V.122. N 12. P. 999. doi 10.1007/s00339-016-0536-3

[27] Shiju E., Siji Narendran N.K., Narayana Rao D., Chandrasekharan K. // Nano Express. 2020. V. 1. N 3. P. 030026. doi 10.1088/2632-959x/abca0f

[28] Chevychelova T.A., Grevtseva I.G., Zvyagin A.I., Smirnov M.S., Ovchinnikov O.V., Ganeev R.A. // Opt. Mater. 2021. V. 111. P. 110583. doi 10.1016/j.optmat.2020.110583

[29] Li J.F. etal. // Nat. Protoc. 2013. V. 8. N 1. P. 52. doi 10.1038/nprot.2012.141

[30] Sheik-Bahae M., Said A.A., Wei T.H., Hagan D.J., Van Stryland E.W. // IEEE J. Quantum Electron. 1990. V. 26. P. 760. doi 10.1109/3.53394

[31] Liu X., Guo S., Wang H., Hou L. // Opt. Commun. 2001. V. 197. P. 431. doi 10.1016/s0030-4018(01)01406-7

[32] Sheik-Bahae M., Hutchings D.C., Hagan D.J., Van Stryland E.W. // IEEE J. Quantum Electron. 1991. V. 27. N 6. P. 1296. doi 10.1109/3.89946

[33] Zhu M., Aikens C.M., Hollander F.J., Schatz G.C., Jin R. // J. Am. Chem. Soc. 2008. V. 130. N 18. P. 5883. doi $10.1021 / j a 801173 r$ 